\title{
Literature information in PubChem: associations between PubChem records and scientific articles
}

\author{
Sunghwan Kim º, Paul A. Thiessen, Tiejun Cheng, Bo Yu, Benjamin A. Shoemaker, Jiyao Wang, Evan E. Bolton, \\ Yanli Wang and Stephen H. Bryant
}

\begin{abstract}
Background: PubChem is an open archive consisting of a set of three primary public databases (BioAssay, Compound, and Substance). It contains information on a broad range of chemical entities, including small molecules, lipids, carbohydrates, and (chemically modified) amino acid and nucleic acid sequences (including siRNA and miRNA). Currently (as of Nov. 2015), PubChem contains more than 150 million depositor-provided chemical substance descriptions, 60 million unique chemical structures, and 225 million biological activity test results provided from over 1 million biological assay records.

Description: Many PubChem records (substances, compounds, and assays) include depositor-provided cross-references to scientific articles in PubMed. Some PubChem contributors provide bioactivity data extracted from scientific articles. Literature-derived bioactivity data complement high-throughput screening (HTS) data from the concluded $\mathrm{NIH}$ Molecular Libraries Program and other HTS projects. Some journals provide PubChem with information on chemicals that appear in their newly published articles, enabling concurrent publication of scientific articles in journals and associated data in public databases. In addition, PubChem links records to PubMed articles indexed with the Medical Subject Heading (MeSH) controlled vocabulary thesaurus.
\end{abstract}

Conclusion: Literature information, both provided by depositors and derived from MeSH annotations, can be accessed using PubChem's web interfaces, enabling users to explore information available in literature related to PubChem records beyond typical web search results.

\section{Background}

PubChem (https://pubchem.ncbi.nlm.nih.gov) [1-6] is an open archive which contains information on a broad range of chemical entities, including small molecules, lipids, carbohydrates, and (chemically modified) amino acid and nucleic acid sequences (including siRNA and miRNA). Since it was launched in 2004 as a component of the Molecular Libraries Program (MLP) of the U.S. National Institutes of Health (NIH), PubChem has been serving as a chemical information resource for scientific

\footnotetext{
*Correspondence: kimsungh@ncbi.nlm.nih.gov
}

National Center for Biotechnology Information, National Library

of Medicine, National Institutes of Health, Department of Health

and Human Services, 8600 Rockville Pike, Bethesda, MD 20894, USA communities in many areas including chemical biology, cheminformatics, and medicinal chemistry.

Data organization in PubChem is described in detail elsewhere [6,7], and only a brief summary is given here. Chemical information contained in PubChem is deposited by more than 350 data contributors including government agencies, academic institutions, pharmaceutical companies, chemical vendors, and publishers. PubChem organizes this information into three primary databases: Substance, Compound, and BioAssay. The Substance database (https://www.ncbi.nlm.nih.gov/pcsubstance) archives depositor-provided chemical substance descriptions. The Compound database (https://www.ncbi.nlm. nih.gov/pccompound) stores unique chemical structures extracted from the Substance database through a 
standardization process. The BioAssay database (https:// www.ncbi.nlm.nih.gov/pcassay) contains descriptions and results of biological assay experiments. The record accessions used for the respective PubChem databases are the Substance ID (SID), Compound ID (CID) and Assay ID (AID).

As of November 2015, PubChem contains more than 150 million depositor-provided substance descriptions, 60 million unique chemical structures, and 225 million biological activity test results (from over 1 million assay experiments performed on more than 2 million smallmolecules covering almost 10,000 unique protein target sequences that correspond to more than 5000 genes). It also contains RNA interference (RNAi) screening assays that target over 15,000 genes. Many of these PubChem records (substances, compounds, and assays) have depositor-provided cross-references to scientific articles in PubMed (https://www.pubmed.gov) [8-11], a biomedical literature search system developed and maintained by the National Center for Biotechnology Information (NCBI) at the National Library of Medicine (NLM), an institute within NIH.

PubMed, whose primary identifier is the PubMed ID (PMID), provides free access to more than 25 million scientific abstracts covering the fields of medicine, nursing, dentistry, veterinary medicine, health care systems, and preclinical sciences. Nearly $90 \%$ of the PubMed contents are from MEDLINE [11, 12], which is the NLM's bibliographic database containing more than 22 million abstracts of journal articles in life sciences with a concentration in biomedicine. A distinctive feature of MEDLINE is that the records are "indexed" with Medical Subject Headings $(\mathrm{MeSH})[13,14]$. MeSH is the NLM's controlled vocabulary thesaurus, consisting of sets of terms naming descriptors in a hierarchical structure. Indexing of scientific papers with $\mathrm{MeSH}$ terms enables users to perform a literature search at various levels of specificity. Of keen interest to PubChem is that MeSH includes a large number of chemical substance concepts, chemical names associated with each concept, and specific/qualified links between these concepts and PMIDs. Considering PubChem contains many chemical names, $\mathrm{MeSH}$ allows PubChem records to be linked to the biomedical literature using matching chemical names.

There are multiple sources of links to PubMed articles within PubChem and different contexts where they can be specified within a given PubChem record type. Understanding where these links can be provided and who provides them can help aid in their use. For example, they can provide PubChem users quick access to the original source article of a bioactivity result. In some cases, data contributors provide chemical information extracted from the scientific literature through manual curation or data mining. In addition, PubChem performs an automated process that annotates PubChem records with $\mathrm{MeSH}$ terms (by means of chemical name matching), creating associations between PubChem records and PubMed articles that share the same MeSH annotation. This work summarizes the various sources of PubMed links in PubChem, explains ways to access these links, and examines the scope of literature information associated with PubChem records.

\section{Construction and content \\ Depositor-provided cross-references to scientific articles Cross-references from substances/compounds to PubMed articles}

Table 1 summarizes the number of depositor-provided cross-references (links) from PubChem substances and compounds to PubMed articles $\left(\mathrm{N}_{\text {SID-PMID }}\right.$ and $\mathrm{N}_{\text {CID- }}$ PMID, respectively) as well as the associated number of substances, compounds, and PubMed article records $\left(\mathrm{N}_{\mathrm{SID}}, \mathrm{N}_{\mathrm{CID}}\right.$, and $\mathrm{N}_{\mathrm{PMID}}$, respectively). As of November 2015, PubChem has more than five million depositorprovided cross-references $\left(\mathrm{N}_{\mathrm{SID}-\mathrm{PMID}}=5,614,567\right)$ from 300 thousand substances $\left(\mathrm{N}_{\mathrm{SID}}=301,358\right)$ to two million PubMed articles $\left(\mathrm{N}_{\mathrm{PMID}}=2,192,601\right)$. Cross-references from compounds to PubMed articles are derived from corresponding substances. Given that compounds are the unique chemical structures extracted from the Substance database (and there can be many substance records for the same chemical), one cross-reference from a compound to a PubMed article is counted if one (or more) of the compound's corresponding substances contains a cross-reference to that given PubMed article. Therefore, the count of compounds with PubMed cross-references is less than the count of substances with cross-references $\left(\mathrm{N}_{\mathrm{CID}}=261,497\right.$ vs. $\left.\mathrm{N}_{\mathrm{SID}}=301,358\right)$. Similarly, the count of CID-PMID cross-references is less than the count of SID-PMID cross-references $\left(\mathrm{N}_{\mathrm{CID} \text {-PMID }}=5,412,256 \mathrm{vs}\right.$. $\left.\mathrm{N}_{\text {SID-PMID }}=5,614,567\right)$.

The biggest source of SID-PMID cross-references is the IBM Almaden Research Center (http://www.research.ibm. com/labs/almaden/index.shtml). This PubChem depositor provides PMIDs of the scientific articles mentioning a particular chemical. While beyond the scope of this paper, they also provide links to patent documents mentioning a particular chemical. The IBM substance records provide more than $90 \%$ of the SID-PMID crossreferences involving about half of all substance records containing cross-references to PubMed articles.

The next largest sources of depositor-provided literature links for PubChem Substance and Compound records are ChemDB [http://chemdb.niaid.nih.gov/provided by the U.S. National Institute of Allergy and Infectious Diseases (NIAID)] and the Comparative 
Table 1 Summary of depositor-provided cross-references to PubMed articles from PubChem substances and compounds

\begin{tabular}{|c|c|c|c|c|c|}
\hline & \multicolumn{2}{|c|}{ Number of cross-references } & \multicolumn{3}{|c|}{ Number of records involved } \\
\hline & $\mathrm{N}_{\text {SID-PMID }}$ & $\mathrm{N}_{\text {CID-PMID }}$ & $\mathrm{N}_{\text {PMID }}$ & $\mathrm{N}_{\text {SID }}$ & $\mathrm{N}_{\mathrm{CID}}$ \\
\hline All & $5,614,567$ & $5,412,256$ & $2,192,601$ & 301,358 & 261,497 \\
\hline IBM Almaden Research Center ${ }^{\mathrm{a}}$ & $5,196,617$ & $5,125,878$ & $2,107,354$ & 152,777 & 147,576 \\
\hline Comparative toxicogenomics database ${ }^{b}$ & 226,585 & 111,029 & 110,000 & 14,463 & 7856 \\
\hline NIAID ChemDBc & 144,477 & 133,012 & 11,951 & 114,953 & 104,418 \\
\hline IUPHAR/BPS guide to PHARMACOLOGYd & 14,309 & 11,250 & 7163 & 6398 & 4913 \\
\hline Human metabolome database $e^{e}$ & 13,998 & 13,971 & 10,414 & 1788 & 1781 \\
\hline Immune epitope database $(\mathrm{IEDB})^{f}$ & 4849 & 3863 & 1747 & 2067 & 1948 \\
\hline $\mathrm{BioCyc}^{\mathrm{g}}$ & 3318 & 3267 & 1386 & 2989 & 2939 \\
\hline DrugBank ${ }^{h}$ & 3249 & 3225 & 3158 & 1044 & 1030 \\
\hline Biocatalysis/biodegradation database (BBD) ${ }^{i}$ & 2299 & 2299 & 644 & 1343 & 1342 \\
\hline Bioinformatics and drug design (BIDD) group & 2270 & 2262 & 91 & 1768 & 1673 \\
\hline Others & 2596 & 2200 & 1180 & 1768 & 1448 \\
\hline
\end{tabular}

$\mathrm{N}_{\text {SID }}$ and $\mathrm{N}_{\mathrm{CID}}$ are the number of PubChem substances and compounds with depositor-provided cross-references to PubMed articles, respectively, and $\mathrm{N}_{\text {SID-PMID }}$ and $\mathrm{N}_{\text {CID-PMID }}$ are the number of depositor-provided cross-references from PubChem substance and compound records to PubMed articles, respectively. $\mathrm{N}_{\mathrm{PMID}}$ is the number of unique PubMed articles associated with the PubChem records via the depositor-provided cross-references

a http://www.research.ibm.com/labs/almaden/index.shtml

b Ref. [15]. http://ctdbase.org

c http://chemdb.niaid.nih.gov

d Ref. [16]. http://www.guidetopharmacology.org

e Ref. [17]. http://www.hmdb.ca

f Ref. [18]. http://www.iedb.org

9 Ref. [19]. http://biocyc.org

h Ref. [20]. http://www.drugbank.ca

i Ref. [21]. http://eawag-bbd.ethz.ch

j http://bidd.nus.edu.sg

Toxicogenomics Database (CTD) (http://ctdbase.org/) [15], which provide 144 thousand cross-references for 114 thousand substances and 226 thousand cross-references for 14 thousand substances, respectively. Other notable contributors for biologically relevant SID-PMID cross-references include IUPHAR/BPS Guide to Pharmacology (http://www.guidetopharmacology.org) [16], Human Metabolome Database (HMDB) (http://www. hmdb.ca/) [17], Immune Epitope Database (IEDB) (http://www.iedb.org/) [18], BioCyc (http://biocyc.org/) [19], DrugBank (http://www.drugbank.ca/) [20], and the Biocatalysis/Biodegradation database (BBD) (http:// eawag-bbd.ethz.ch/) [21].

The disproportionate number of cross-references and chemical substance coverage coming from a handful of data contributors (Table 1) points to their diverging focus areas in data collection. For example, IBM uses data mining to collect information on patents and scientific papers that mention a very broad range of chemicals, resulting in a large number of links to scientific papers. On the other hand, DrugBank primarily focuses on U.S. Food and Drug Administration (FDA) drugs (approved and experimental) and collects relevant information through manual curation. Similarly, the IEDB resource performs manual curation but considers chemical substances in the immunology epitope research domain. As such, PMID cross-references provided by different chemical substance data depositors can have a significantly varied focus and approach.

\section{Bioactivity data extracted from scientific articles}

Data contributors may supply PubChem with a list of PMIDs for scientific articles that have information relevant to a given assay record. These articles may provide various kinds of information related to the assay, such as assay detection method, experimental protocols, assay targets, diseases associated with the targets, known ligands that bind to the targets, and so on. These articles may or may not be relevant to substances and compounds tested in those assays. With that said, it is of particular interest that some PubChem contributors provide bioactivity information extracted from the literature through the means of manual curation or data mining. These data are an important source of bioactivity information in PubChem that complements high-throughput screening (HTS) data from the now-concluded NIH Molecular 
Libraries Program. Table 2 shows major contributors that provide literature-extracted bioactivity data.

ChEMBL (https://www.ebi.ac.uk/chembl/) [22] is a public database of bioactivity information for compounds. Most of the bioactivity data contained in ChEMBL is manually extracted from the full text of peer-reviewed scientific papers published in about 50 journals in the medicinal chemistry and natural product domains. From each paper, detailed information is extracted on the compounds tested, the assays performed, and the targets for the assays. Often multiple data sets are generated for a single paper, resulting in multiple BioAssay records for that publication. The ChEMBL data is updated on a regular basis, with periodic releases approximately every 3-6 months. The current version of ChEMBL (ChEMBL v20, as of September 2015) contains 13 million bioactivities for 1.5 million unique compounds, abstracted from about 60 thousand publications or provided by other data sources. ChEMBL contributes its literature-derived data to the PubChem BioAssay database.

The Guide to Pharmacology (http://www.guidetopharmacology.org) [16], created under the auspices of the International Union of Basic and Clinical Pharmacology (IUPHAR) and the British Pharmacological Society (BPS), provides in-depth integrated views of the pharmacology, genetics, functions, and pathophysiology of important drug targets, including G-protein-coupled receptors (GPCRs), ion channels, and nuclear hormone receptors (NHRs). In addition, it provides information on the interactions between these target proteins and their ligands. PubMed citations containing the bioactivity data for these ligands are also provided to PubChem.

The PDBbind database (http://www.pdbbind-cn.org) [23] collects experimentally measured binding affinity data $\left(\mathrm{IC}_{50}, \mathrm{~K}_{\mathrm{d}}\right.$, or $\left.\mathrm{K}_{\mathrm{i}}\right)$ for biomolecular complexes in the Protein Data Bank (PDB) [24]. The majority of the data in PDBbind is for complexes between proteins and small molecule ligands, although it also contains other types of complexes such as protein-protein, proteinnucleic acid, and nucleic acid-ligand complexes. All binding affinity data in PDBbind are manually curated from nearly 24,000 original references. PDBbind contributes to PubChem binding affinity data for $\sim 10,000$ protein-ligand complexes that involve $\sim 3000$ unique small molecule chemical structures, with related information including the PMID for the source article, PDB ID and MMDB ID [25] for the protein-ligand structures, and the protein GI and name for the protein target.

BindingDB (https://www.bindingdb.org) [26] provides measured binding affinities, focusing chiefly on the interactions of proteins considered to be drug targets with small, drug-like molecules. BindingDB contains more than one million binding interactions for seven thousand protein targets and 495 thousand small molecules.

GLIDA (GPCR-Ligand Database) (http://pharminfo. pharm.kyoto-u.ac.jp/services/glida/) [27] is a public chemical genomics database that provides information on GPCRs, their ligands, and interactions between them. The ligand-binding information was manually collected and curated from PubMed and other sources. Although GLIDA has ceased updates since 2010, it is still operational and its data are fully accessible.

In addition to these resources containing manually curated sources of bioactivity, there is a growing number of AID-PMID references deposited directly by the authors of scientific publications uploading their research data into the PubChem BioAssay database. This includes researchers from RNAi screening and is open to all researchers in the chemical biology, medicinal chemistry, and related fields. Such submissions help to satisfy

Table 2 Summary of cross-references from literature-extracted bioassay data to PubMed articles

\begin{tabular}{|c|c|c|c|c|c|c|c|}
\hline Source & $\mathrm{N}_{\text {AID-PMID }}$ & $\mathrm{N}_{\text {SID-PMID }}$ & $\mathrm{N}_{\text {CID-PMID }}$ & $\mathrm{N}_{\text {PMID }}$ & $\mathrm{N}_{\text {AID }}$ & $\mathrm{N}_{\text {SID }}$ & $\mathrm{N}_{\mathrm{CID}}$ \\
\hline ChEMBL $^{a}$ & 829,503 & $1,068,347$ & $1,058,637$ & 55,582 & 828,594 & 860,191 & 849,149 \\
\hline PDBbind $^{b}$ & 6946 & 20,221 & 16,993 & 5252 & 4 & 10,543 & 8158 \\
\hline IUPHAR/BPS guide to pharmacology ${ }^{c}$ & 442 & 1088 & 1080 & 151 & 55 & 273 & 264 \\
\hline Binding DB $^{d}$ & 143 & 3114 & 3113 & 121 & 19 & 3101 & 3098 \\
\hline GLIDA $^{e}$ & - & - & - & - & 6 & 19,474 & 19,458 \\
\hline
\end{tabular}

$\mathrm{N}_{\mathrm{AID}}, \mathrm{N}_{\mathrm{SID}}$ and $\mathrm{N}_{\mathrm{CID}}$ are the number of PubChem assays, substances and compounds extracted from scientific articles, respectively; and $\mathrm{N}_{\text {AID-PMID, }} \mathrm{N}_{\text {SID-PMID }}$ and $\mathrm{N}_{\mathrm{CID}-\mathrm{PMID}}$ are the number of cross-references from PubChem assays, substances, and compounds to PubMed articles, respectively. $\mathrm{N}_{\mathrm{PMID}}$ is the number of unique PubMed articles from which the assay data are extracted

a Ref. [22]. https://www.ebi.ac.uk/chembl/

b Ref. [23]. http://www.pdbbind-cn.org

c Ref. [16]. http://www.guidetopharmacology.org

${ }^{d}$ Ref. [26]. https://www.bindingdb.org

e Ref. [27]. http://pharminfo.pharm.kyoto-u.ac.jp/services/glida/ 
open access and data sharing requirements from research funding agencies.

Note that different PubChem BioAssay data sources can provide PubMed links in different ways. For example, ChEMBL provides the cross-reference between an assay and the article from which bioactivity information for that assay were extracted, and therefore, literature evidence on bioactivity data for substances tested in that assay can be deduced from the AID-PMID cross-reference. PDBbind provides information on the source article of bioactivity data for each substance tested in an assay (as a property of the tested substance reported in the assay data table). GLIDA does not explicitly provide literature information for assays or tested substances in them. Instead, it provides the GLIDA ID (the database identifier used in GLIDA) for a substance. This external ID is used to search for the corresponding record in GLIDA to get information on the source article.

\section{Chemical information provided by journal publishers}

Publishers are also an indirect source of records in PubChem. While many funding agencies mandate that data from studies they support should be freely available to the public, some journals also require data submission of research data to public databases as a precondition to publishing a research paper. Data sharing requirements by journals and granting agencies may be satisfied by use of the PubChem data archiving platform.

Some journals directly provide PubChem with literature information for chemicals. Examples include journals published by the Nature Publishing Group (including Nature Chemical Biology, Nature Chemistry, and Nature Communications). When a new article is published in one of these journals, the publisher identifies chemicals studied in the article and submits them to PubChem with information on the source article (including the journal name, authors, title, publication date, and digital object identifier (DOI)). These chemicals are stored in the Substance database, and the substance record page for each chemical has a link to the source article (see Fig. 1). Similarly, on the web page of the original article the publisher provides links to the corresponding substance records in PubChem. This allows readers to readily access comprehensive information available in PubChem for these chemicals, through the compound records associated with the substance records. This demonstrates the benefit of concurrent publication of scientific articles in journals and associated data in public databases.

\section{Automated annotations of PubChem records with PubMed articles via MeSH}

PubChem generates CID-PMID associations via MeSH using depositor-provided chemical names. This is achieved by first determining which depositor-provided chemical names in PubChem are also found in $\mathrm{MeSH}$. Not all chemical names in PubChem are used in this step. Chemical names provided to PubChem are passed through a sort-of crowd-sourcing filtering process, where consistency of chemical name-structure associations of depositor-provided synonyms is determined using differing levels of chemical structure sameness (e.g., potentially grouping different charge states or salt forms of the same chemical). This reduces the count of PubChem Compound records associated with a given chemical name. The resulting filtered chemical names are considered "filtered synonyms" in PubChem.

Once PubChem chemical names are matched to $\mathrm{MeSH}$ concepts, the MeSH concept is associated with the PubChem Compound record containing matching chemical names. Additional consistency filtering suppresses multiple $\mathrm{MeSH}$ concepts from being associated with the same PubChem Compound record (by taking the consensus $\mathrm{MeSH}$ concept for a given compound record). This helps to reduce errors but also helps to provide a consistent chemical representation for a given $\mathrm{MeSH}$ chemical concept.

The CID-PMID associations are generated in a final step. PubMed records that are annotated with a given $\mathrm{MeSH}$ chemical concept are then associated with the PubChem Compound record linked to the MeSH chemical concept. In this way (CID $\rightarrow \mathrm{Name} \rightarrow \mathrm{MeSH} \rightarrow$ PMID), linking between PubChem and PubMed is achieved.

The $\mathrm{MeSH}$ annotations of PubChem records are "indexed" as indices for text search within NCBI's Entrez search system [1, 28, 29]. Entrez is the text/numeric search and retrieval system that integrates PubChem's three primary databases (BioAssay, Compound, and Substance) with approximately forty other NCBI databases, including PubMed and MeSH. The Entrez indices are tied to individual records in PubChem and include information on particular aspects (often referred to as fields) of the records. The available fields and their indexed terms in PubChem can be explored on the Advanced Search page as part of the Search Builder (see Fig. 2).

The Entrez indices derived from $\mathrm{MeSH}$ annotations are summarized in Table 3. The "Complete MeSH Term" Entrez index retrieves compounds annotated with the $\mathrm{MeSH}$ term that exactly matches the query, while the "MeSH Term" index searches for those with the MeSH terms that partially match the query. The "MeSH Tree Node" index finds compounds annotated with the query $\mathrm{MeSH}$ term and those with any MeSH terms that correspond to the child nodes beneath the node for the query term. The "MeSH Description" index searches the descriptions of MeSH terms for the query string.

Some MeSH annotations (such as solvents, carcinogens, inhibitors, and so on) are too general to describe a specific 


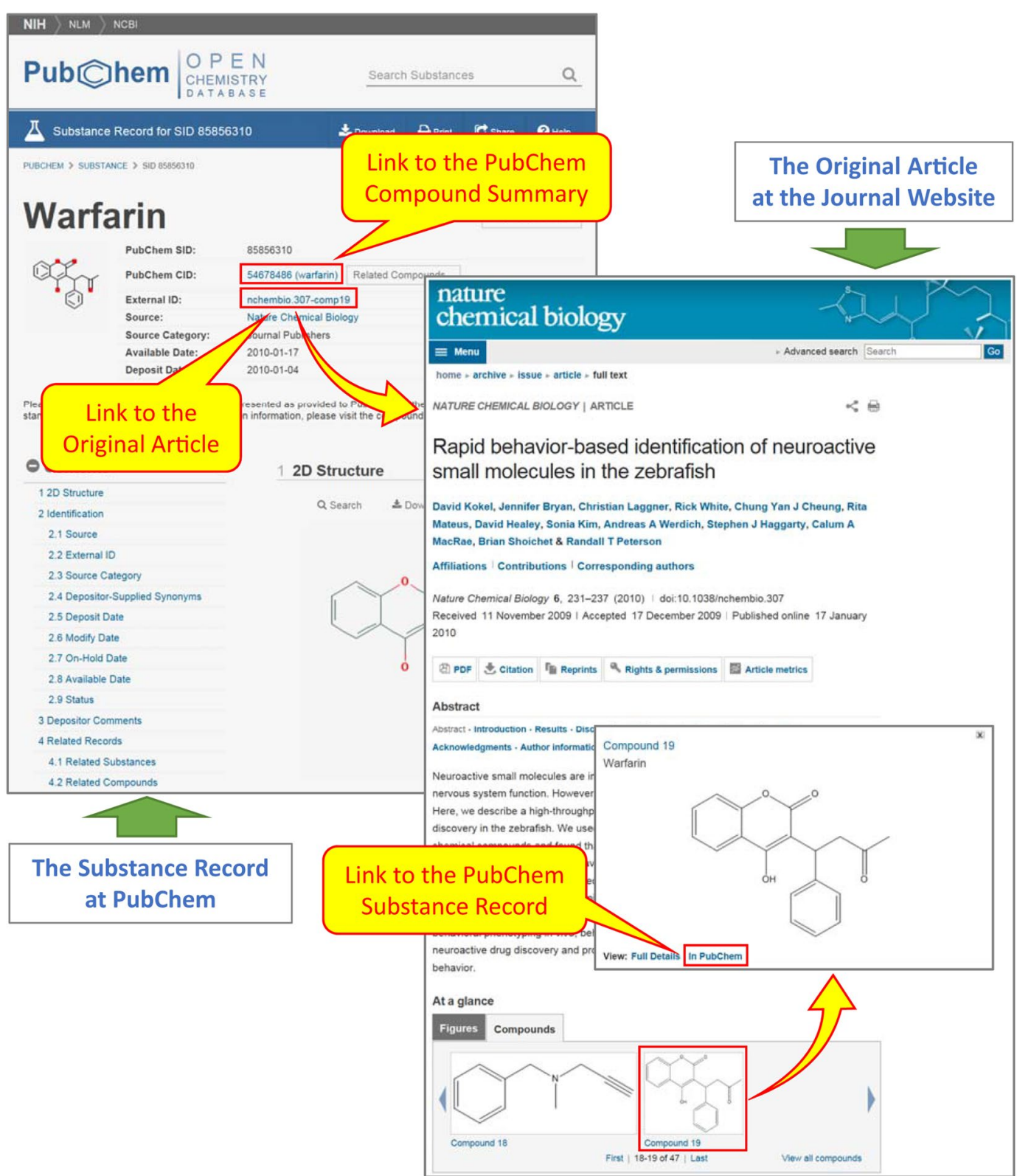

Fig. 1 The substance record page for SID 85856310 (warfarin), with a link to the source article published in Nature Chemical Biology. The original article has a link to SID 85856310 in PubChem, allowing article readers to access comprehensive information on warfarin available in the PubChem Compound database, by clicking a link to the Compound Summary page for CID 54678486

biological function of a compound. Therefore, "Pharmacological Actions" (a subset of MeSH terms) are separately included as an Entrez index, as these annotations indicate specific biological roles of chemical concepts. The "Pharmacological Action ID" index searches for records with the Pharmacological Action annotation corresponding to a numeric identifier assigned by MeSH given as a query.

$\mathrm{MeSH}$ annotations of a compound can be used to annotate substance records corresponding to that compound.
PubChem annotates substance records with MeSH terms for Entrez searching purposes similar to compound records. However, no attempt is made to filter chemical names or MeSH concepts for substance records, using chemical name information directly provided by individual data contributors for a given substance. In addition, the $\mathrm{MeSH}$ annotations of substances are not displayed on the substance record page, to avoid confusion about the provenance of displayed information. 


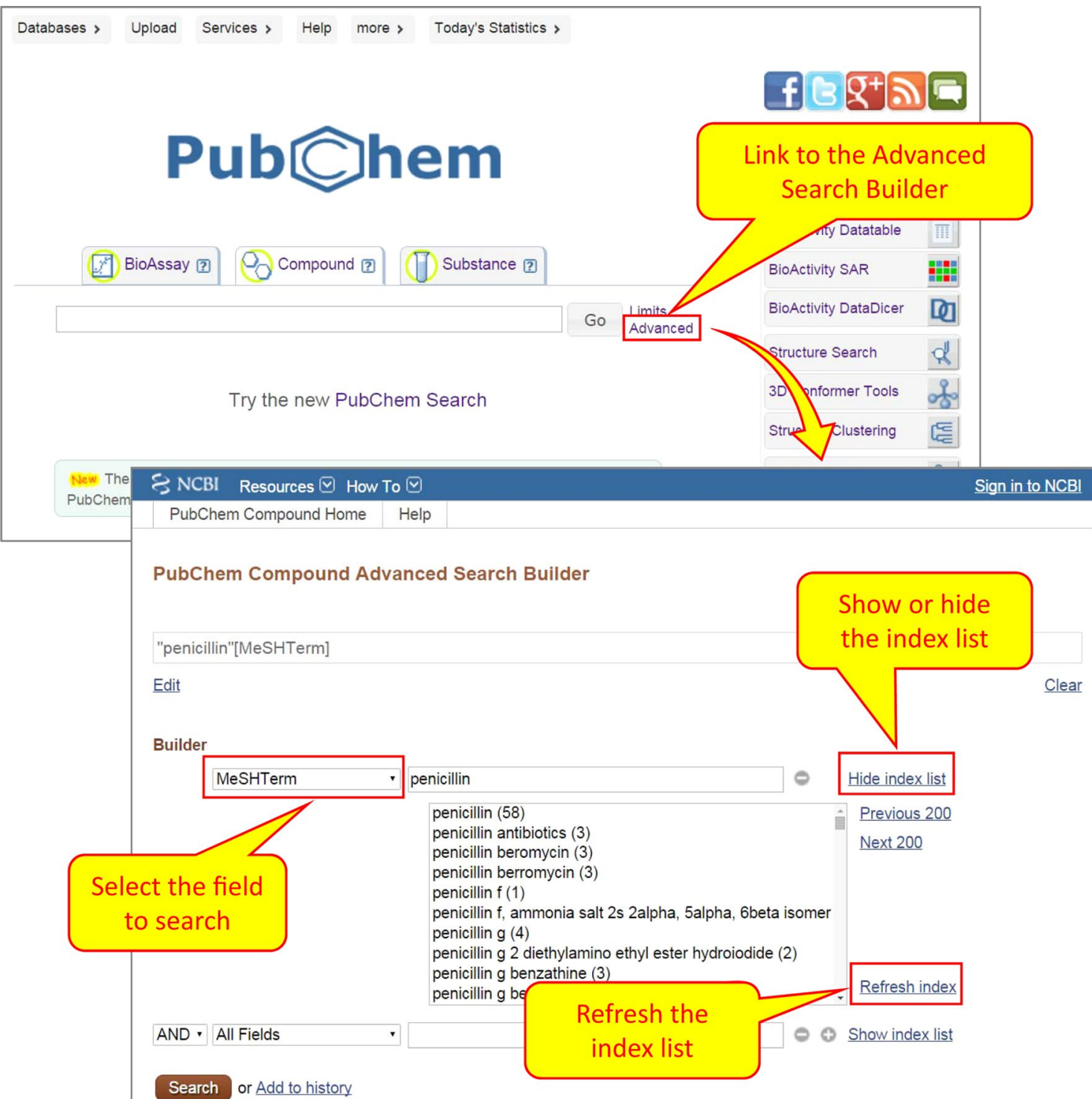

Fig. 2 Retrieving compound records annotated with MeSH terms using the Advanced Search Builder. Clicking the "Advanced" link under the "Compound" tab on the PubChem Homepage directs users to the PubChem Compound Advanced Search Builder. Selecting the "MeSHTerm" from the dropdown menu and providing a MeSH term in the search box will retrieve compounds annotated with that MeSH term

$\mathrm{MeSH}$ annotations to compound records made by PubChem are used to annotate assay records testing the compounds. They are also used to generate Entrez indices that allow one to search the PubChem BioAssay database for assays that have tested compounds with a particular MeSH annotation. For example, the "MeSH Term Tested" index retrieves assay records in which any tested compound is annotated with the query $\mathrm{MeSH}$ term. The "MeSH Term Active" retrieves assays in which only active compounds are annotated with the query $\mathrm{MeSH}$ term.

\section{Utility and discussion}

Entrez links from PubChem records to PubMed articles

The associations between PubChem records and PubMed records, whether provided by data contributors or computationally derived via common $\mathrm{MeSH}$ annotations, are available to PubChem users as Entrez links. In general, Entrez links are crosslinks between records in different Entrez databases or within the same Entrez database. The Entrez links provide a way to discover relevant information in Entrez databases based on a user's specific interests. For example, one can readily obtain a list of 
Table 3 Entrez indices used to search for records with MeSH annotations

\begin{tabular}{|c|c|}
\hline Entrez index & Description \\
\hline \multicolumn{2}{|l|}{ Compound database } \\
\hline Complete MeSH term & Retrieve compounds annotated with the MeSH term that exactly matches the query \\
\hline MESH term & $\begin{array}{l}\text { Retrieve compounds annotated with MeSH terms that partially match the query. Note that MeSH entry terms (synonyms for } \\
\text { the Medical Subject Heading term) are also indexed }\end{array}$ \\
\hline MeSH tree node & $\begin{array}{l}\text { Retrieve compounds annotated with the MeSH term that match the query and those annotated with any MeSH terms } \\
\text { beneath the node corresponding to that MeSH term. For example, "Penicillins[MeSHTreeNode]" will retrieve records anno- } \\
\text { tated with MeSH term "Penicillins"and those with MeSH terms "Oxacillin", "Cloxacillin", and so on, which correspond to child } \\
\text { nodes beneath the "Penicillins" node in the MeSH tree }\end{array}$ \\
\hline MeSH description & Retrieve compounds annotated with the MeSH terms whose description contains the query string \\
\hline PharmAction & Retrieve compounds annotated with the Pharmacological Action term, which are a subset of MeSH terms \\
\hline PharmActionID & $\begin{array}{l}\text { Retrieve compounds annotated with the Pharmacological Action term corresponding to the numeric identifier given as a } \\
\text { query }\end{array}$ \\
\hline \multicolumn{2}{|l|}{ BioAssay database } \\
\hline MeSH term active & Retrieve assays in which only an active substance is annotated with the MeSH term given as a query \\
\hline MeSH term tested & Retrieve assays in which any tested substance is annotated with the MeSH term given as a query \\
\hline MeSH description active & Retrieve assays in which only an active substance is annotated with the MeSH terms whose descriptions have a query string \\
\hline MeSH description tested & Retrieve assays in which any tested substance is annotated with the MeSH terms whose descriptions have a query string \\
\hline Pharm action active & Retrieve assays in which only an active substance has the Pharmacological Action annotation given as a query \\
\hline Pharm action tested & Retrieve assays in which any tested substance has the Pharmacological Action annotation given as a query \\
\hline
\end{tabular}

scientific articles related to a particular molecule through an Entrez link from the corresponding compound record in PubChem to associated articles in PubMed. As another example, users may access all data sets (AID) in the BioAssay database associated with a research article in PubMed. Entrez links from PubChem to PubMed records are available to users in several ways.

\section{Entrez links on the document summary (DocSum) page}

An Entrez document summary (DocSum) page, as shown in Fig. 3, displays multiple records returned from an Entrez search. Entrez links are available via the "Find related data" menu on the right column. If no compounds are selected, these links will be applied to the entire search result list by default. Note that, when "PubMed" is selected from the Database drop-down menu, three items appear under the "Option" menu: "PubMed Citations", "PubMed (MeSH Keyword)", and "PubMed (Publisher)". These options correspond to different types of Compound-to-PubMed Entrez Links.

The first option "PubMed Citations" is for Entrez links derived from cross-references to PubMed articles that data contributors provided to PubChem. That is, this option will retrieve scientific articles containing information relevant to a given chemical, according to PubChem depositors. On the other hand, the third option, "PubMed (Publisher)", is from CID-PMID associations provided by journal publishers to PubMed (not PubChem) as a part of PubMed abstract submission. Both the first and third options are similar in the sense that some organization (e.g., data contributors or journal publishers) contributed the chemical-literature associations. However, the second option "PubMed (MeSH Keyword)" corresponds to CID-PMID associations derived via $\mathrm{MeSH}$ annotations. This option returns articles annotated by Medline indexers with MeSH terms (chemical names). MeSH annotation can be further leveraged to find which articles have a given $\mathrm{MeSH}$ term as a major topic to obtain articles specifically about that chemical.

Three different types of Entrez Links also exist for substance records to PubMed articles, similar to the Compound-to-PubMed links. For the BioAssay-to-PubMed links, only one type of Entrez link to PubMed is available through the right column of the DocSum page. This returns publications associated with the BioAssay records, based on AID-PMID associations provided by data contributors to PubChem. These publications may be either source articles from which assay data are collected in curation projects, or general articles that provide background information relevant to the assay.

\section{Entrez links on the Compound Summary page}

The Compound Summary page of a compound provides an aggregated view of all available information on that compound collected from various data sources (Fig. 4). When a compound has literature information, this will be shown in the Literature section. A link to PubMed articles associated with the compound via depositor-provided cross-references is displayed under the Depositor Provided PubMed Citations section, and a link to those 
Display Settings: $\nabla$ Summary, 20 per page, Sorted by Default order

Results: 1 to 20 of 60

$\square$

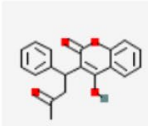

2.

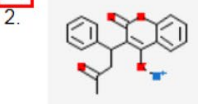

Check boxes to retrieve
PubMed articles associated

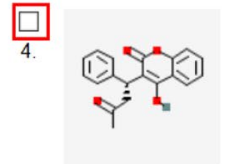

(R)-Warfarin; Dextrowarfarin; (R)-(+)-Warfarin ...

MW: $308.327940 \mathrm{~g} / \mathrm{mol}$ MF: $\mathrm{C}_{18} \mathrm{H}_{10} \mathrm{O}_{4}$

IUPAC name: 4-hydroxy-3-[(1R)-3-oxo-1-phenylbutyl]chromen-2-one

Create Date: $2011-12-26$

CID: 54684598

Summary Similar Compounds Same Parent, Connectivity Mixture/Component Compounds Active in 2 of 41 BioAssays
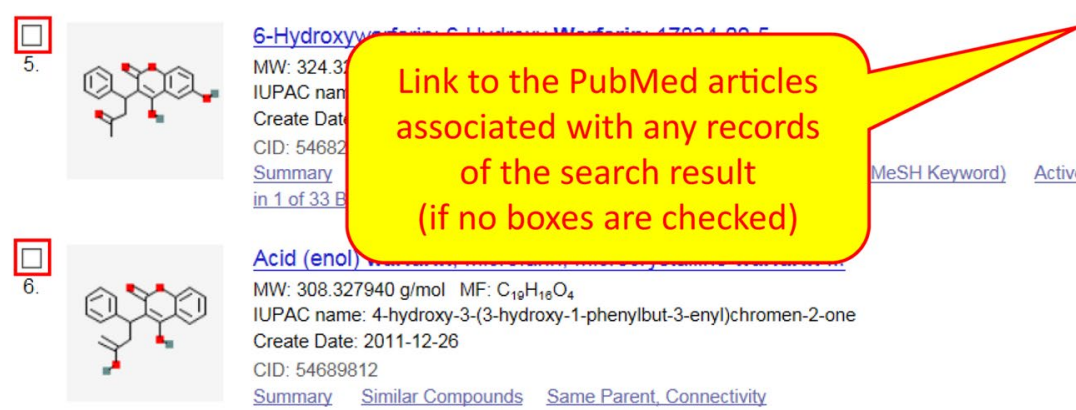

Link to the PubMed articles associated with a single record MW: $308.327940 \mathrm{~g} / \mathrm{mol} \quad \mathrm{MF}: \mathrm{C}_{18} \mathrm{H}_{18} \mathrm{O}_{4}$ Creacte: 4-hydroxy-3-3 CID: 54678486 Similar Compothlos Same Parent, Connectivity Mixture/Component

Warfarin sodium; Sodium warfarin; Jantoven ... MW: $330.309769 \mathrm{~g} / \mathrm{mol}$ MF: $\mathrm{C}_{18} \mathrm{H}_{15} \mathrm{NaO}_{4}$ IUPAC name: sodium;2-oxo-3-(3-oxo-1-phenylbutyl)chromen-4-olate
Create Date: 2007-07-11

Similar Compounds Same Parent, Connectivity
Filters: $\underline{\text { Manage Filters }}$

Actions on your results BioActivity Analysis Analyze the BioActivities of the compounds

Structure Clustering

Cluster structures based on structural similarity

Structure Download Download the structures in various formats

Refine your results - What's this?

Chemical Properties

Rule of 5 (44)

BioActivity Experiments

BioAssays, Active (15)

BioAssays, Tested (25)

Protein 3D Structures (1)

AT Structure Of Human Cytochrome P450 Cyp2c9 (1)

BioMedical Annotation

Pharmacological Actions (9)

$\boxplus$ Anticoagulants (9)

Depositor Category

Chemical Vendors (47)

NIH Molecular Libraries (13)

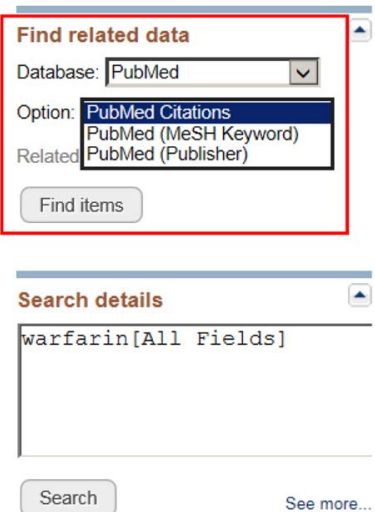

Fig. 3 The document summary (DocSum) page that shows the results for a search for "warfarin". Scientific articles associated with the returned compound records can be accessed via the Entrez Links, which are available under the "Find related data" menu (for multiple records) or from a link for individual compound records

associated with the compound via common MeSH annotations is shown under the NLM Curated PubMed Citations section. In some cases, the per compound PMID associations derived via $\mathrm{MeSH}$ annotations are categorized according to $\mathrm{MeSH}$ subheadings, which allow for describing particular aspects of a subject. MeSH terms associated with a compound are displayed under the Classification section.

\section{Entrez filters from PubChem records to PubMed articles}

One may want to retrieve all PubChem records associated with PubMed articles. This can be done using Entrez filters, which indicate whether or not a given record in an Entrez database has a particular type of annotations. The Entrez filters may be used to subset other Entrez searches according to this annotation type, by adding the filter to the query string. Importantly, Entrez filters are closely 


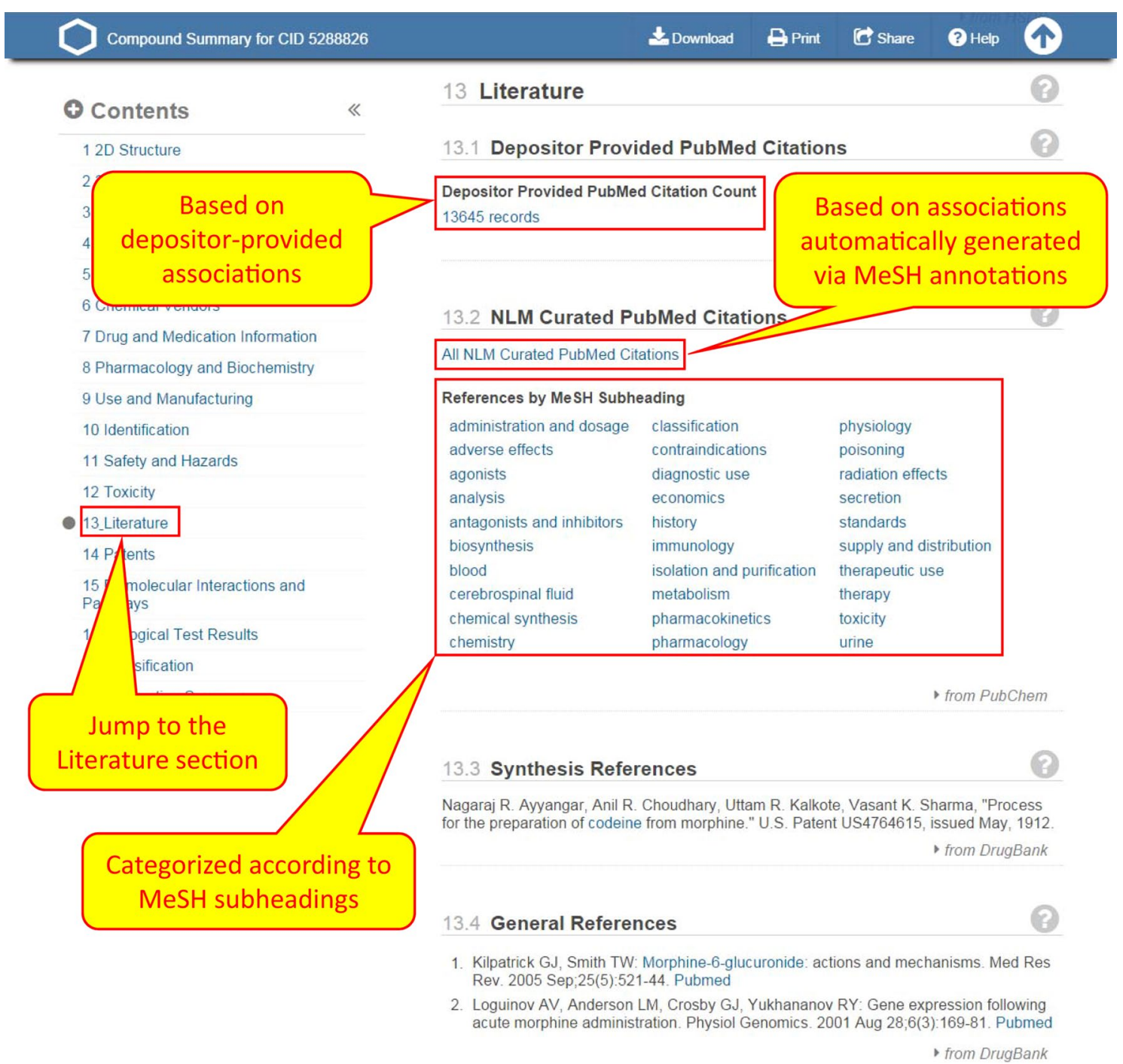

Fig. 4 The literature section of the Compound Summary page (DocSum) for CID 5288826 (morphine). Clicking the "Literature" section in the Table of Contents allows users to jump to the literature section, which consists of two subsections: depositor-provided and NLM-curated PubMed citations

related to Entrez links in that many Entrez filters are generated by checking whether a given record in an Entrez database has an Entrez link to a record in the same or different Entrez database. The filters for each Entrez database may be listed by going to the advanced search page of each database, selecting "Filter" from the "All Fields" dropdown, and clicking "Show index list" (Fig. 5).

Table 4 lists the Entrez Filters available in PubChem that can be used to get the list of records associated with PubMed articles. As mentioned in the previous section, a DocSum page for a compound search result (Fig. 3) shows three different Entrez links between compounds and PubMed articles. These three links are used to generate three Entrez filters that indicate the presence of associated PubMed articles for compound records: (1) pccompound_pubmed, (2) pccompound_pubmed_ mesh, and (3) pccompound_pccompound_publisher. The "pccompound_pubmed" filter allows one to retrieve compound records with cross-references provided to PubChem by data contributors. The "pccompound_pubmed_publisher" filter retrieves those with cross-references to PubMed articles that are provided to PubMed by publishers. The "pccompound_pubmed_mesh" filter retrieves those with computationally generated links to PubMed articles that have a common MeSH annotation, as explained in the previous section. 


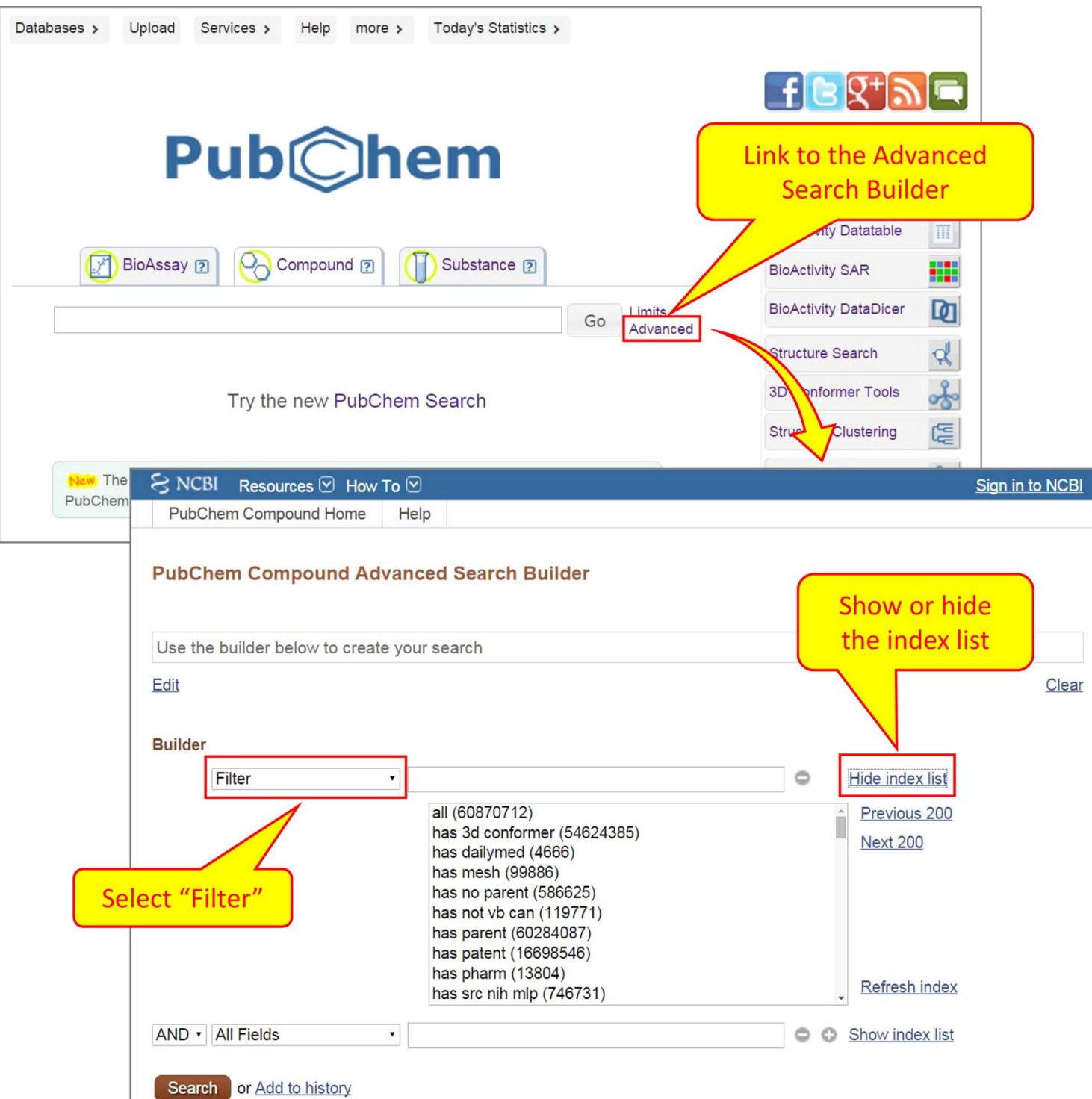

Fig. 5 Retrieving compound records with a particular property using the Entrez filters. Selecting the "Filter" option under the drop-down menu on the PubChem Compound Advanced Search Builder allows you to retrieve compounds with a particular property or annotation. Available filters can be shown or hidden by clicking the "Show/Hide index list" button

While the "pccompound_mesh" filter retrieves any compounds with $\mathrm{MeSH}$ annotations, the "pccompound_mesh_ pharm" filter selects those with Pharmacological Action annotations. These filters are equivalent to the "has_mesh" and "has_pharm" filters, respectively. The "pccompound_ pmc" filter allows one to choose compounds that have associated full-text articles in PubMed Central.

The Substance database also has Entrez filters that are similar to those for compound records listed in Table 4. For example, the "pcsubstance_pubmed" and "pcsubstance_pubmed_publisher" filters can be used to find substance records with cross-references to PubMed that are provided by PubChem depositors to PubChem and by publishers to PubMed, respectively.

\section{Programmatic access to literature information in PubChem} Literature information in PubChem can be programmatically accessed through E-Utilities [30] or PUG-REST [31]. More detailed information on programmatic access to PubChem is given elsewhere [31]. 
Table 4 Entrez filters used to search for records with MeSH annotations

\begin{tabular}{|c|c|}
\hline Entrez filter & Description \\
\hline \multicolumn{2}{|l|}{ Compound database } \\
\hline has_mesh & Equivalent to "pccompound_mesh" \\
\hline has_pharm & Equivalent to "pccompound_mesh_pharm" \\
\hline pccompound_mesh & Select compounds annotated with MeSH terms. Equivalent to "has_mesh" \\
\hline pccompound_mesh_pharm & Select compounds annotated with MeSH Pharmacological Actions. Equivalent to "has_pharm" \\
\hline pccompound_pmc & Select compounds that have associated full-text articles in PubMed Central \\
\hline pccompound_pubmed & Select compounds that have depositor-provided cross-references to PubMed articles \\
\hline pccompound_pubmed_mesh & Select compounds associated with PubMed abstracts that are annotated with common MeSH annotations \\
\hline pccompound_pubmed_publisher & Select compounds that have cross-references to PubMed articles, provided to PubMed by publishers \\
\hline \multicolumn{2}{|l|}{ BioAssay database } \\
\hline pcassy_pmc & Select assays that have associated full-text articles in PubMed Central \\
\hline pcassay_pubmed & Select assays that have depositor-provided cross-references to PubMed articles \\
\hline pcassay_pubmed_major & $\begin{array}{l}\text { Select assays that have cross-references to the PubMed articles that contains the original bioactivity data in } \\
\text { the assays }\end{array}$ \\
\hline
\end{tabular}

\section{Discussion}

Figure 6 shows the frequencies of PMIDs per CID and CIDs per PMID for the three types of CID-PMID associations: those provided to PubChem by PubChem depositors (pccompound_pubmed), those derived by PubChem through matching between chemical names and $\mathrm{MeSH}$ terms (pccompound_pubmed_mesh), and those provided to PubMed by journal publishers (pccompound_pubmed_publisher). Note that the frequencies for the pccompound_pubmed_publisher links are much lower than the other two link types because only a small number of publishers submit CID-PMID associations to PubMed. Therefore, this section focuses the other two link types.

The majority of compounds have no more than ten associated PMIDs (i.e., $95 \%$ of CIDs with depositorprovided PMIDs and $70 \%$ of CIDs with automated annotations via $\mathrm{MeSH}$ ). However, some compounds are associated with many PMIDs. For example, D-glucose has as many as 130,545 depositor-provided PMIDs and 132,017 PMIDs generated via MeSH.

The Venn diagrams shown in Fig. 7 illustrate the overlap between CID-PMID associations provided by depositors and derived annotations via $\mathrm{MeSH}$. Among the 39.2 million CID-PMID associations, only $4 \%$ overlap, being both depositor-provided and NLMcurated, indicating that the two types of associations are nearly orthogonal and complement each other. Of these $4 \%$ overlapping CID-PMID pairs, the CIDs and PMIDs that make them up correspond to 10 and $19 \%$ of all CIDs and all PMIDs found in all CID-PMID pairs, respectively. Importantly, although depositor-provided
CID-PMID links are much fewer in number than those generated via $\mathrm{MeSH}$, there are more CIDs involved in the depositor-provided links than in those derived via $\mathrm{MeSH}$.

It is worth noting that the CID-PMID associations via $\mathrm{MeSH}$ tend to be limited to chemicals that are sufficiently well known to be included in MeSH. These chemicals correspond to only a small fraction of all chemicals studied in scientific articles contained in PubMed. Therefore, associations generated via $\mathrm{MeSH}$ may ignore specific chemicals (favoring instead a chemical class) unless they are already included in $\mathrm{MeSH}$ or sufficiently noteworthy to be specifically added. With that said, depositorprovided CID-PMID associations do not suffer the same limitation as $\mathrm{MeSH}$ and provide a greatly increased number of chemicals mentioned in the biomedical literature, as suggested in Fig. 7.

However, a caveat against depositor-provided associations is that the accuracy and usefulness of these links solely rely on individual depositors' quality control efforts, as PubChem does not (currently) provide an independent quality control mechanism. To address this, PubChem is exploring a few possibilities. For example, one option is to process PubMed abstracts (and PubMed Central full-text papers) using text-mining software for chemical recognition (in text, tables, and figures). The resulting CID-PMID associations can be used to crossvalidate the existing depositor-provided associations and to better understand the context of the chemical mention. Annotation of CID-PMID associations (e.g., location, context) may help prioritize the display of PMIDs associated to a given compound. 

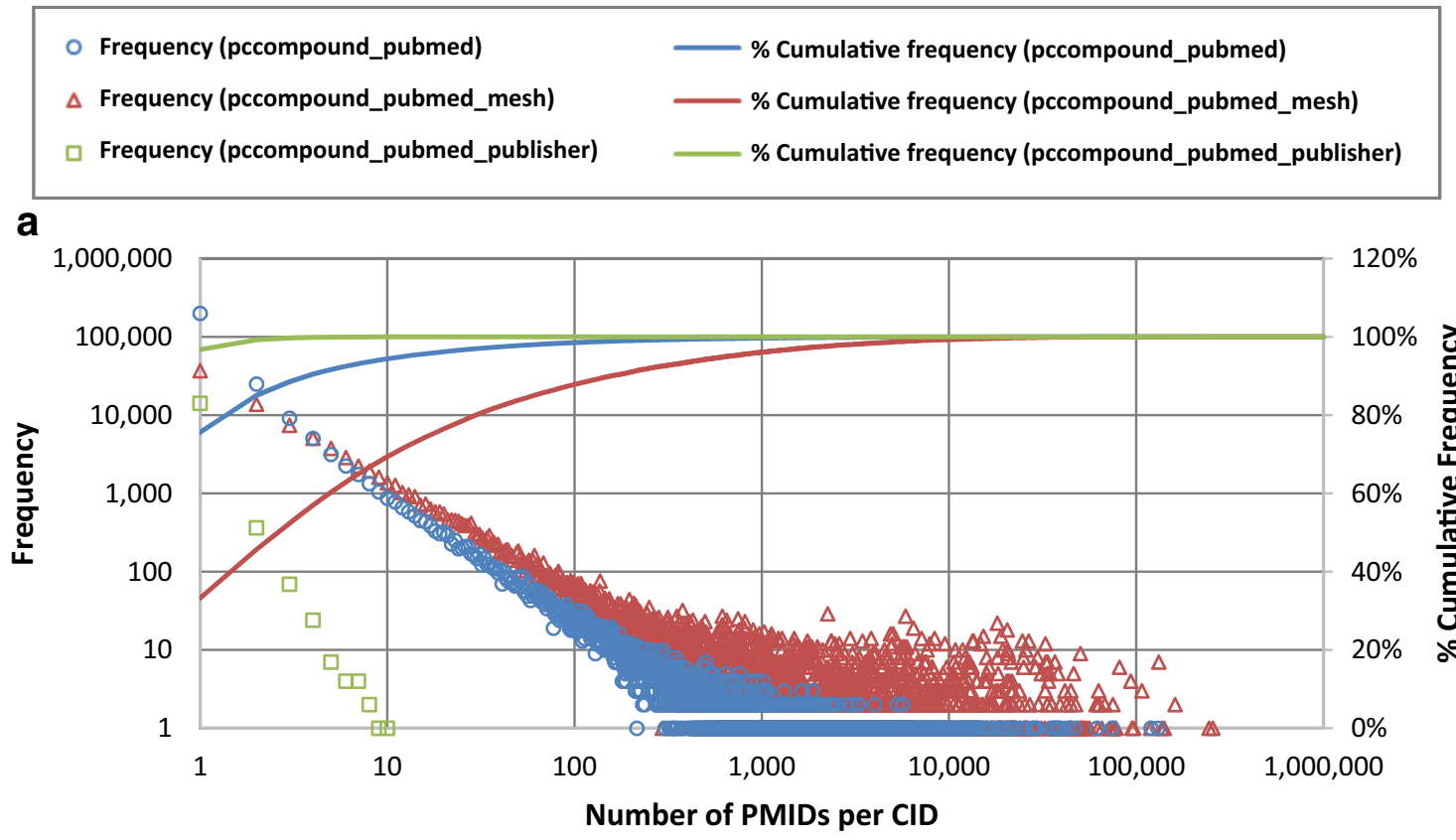

b

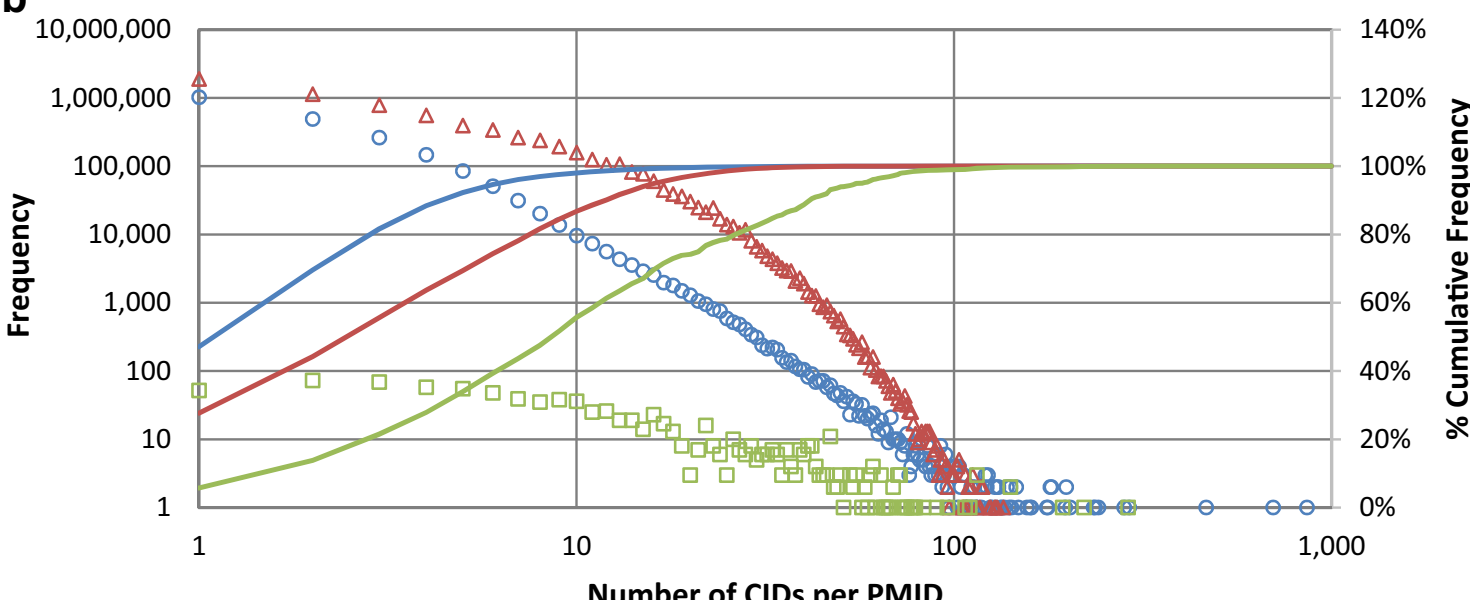

Fig. 6 Distribution of PMIDs per CID and CIDs per PMID for three types of Entrez links. Distributions of $\mathbf{a}$ PMIDs per CID and $\mathbf{b}$ CIDs per PMID are shown for three Entrez links between PubChem Compound and PubMed:"pccompound_pubmed", "pccompound_pubmed_mesh", and "pccompound_pubmed_publisher". See texts for the description of these links

\section{Conclusions}

Literature information available in PubChem for substances, compounds and assays, as well as how this information can be accessed, was described. Individual data contributors provide PubChem with cross-references between chemical substances and PubMed articles that contain information on that substance. From these SID-PMID cross-references, PubChem generates crossreferences between the corresponding compound and the PubMed articles (i.e., CID-PMID cross-references). Data contributors can also supply a list of PMIDs for scientific articles that have information relevant to a given assay record. These articles may contain various kinds of information related to the assay, including experimental protocols, assay targets, diseases associated with the targets, and known ligands that bind the targets. Of particular interest, some data contributors provide bioactivity data extracted from literature through manual curation or data mining and are an important source of bioactivity information in PubChem that complement HTS data from the now-concluded NIH Molecular Libraries Program and other HTS projects. In addition to communityprovided literature information, PubChem generates Entrez links between PubChem records and PubMed 


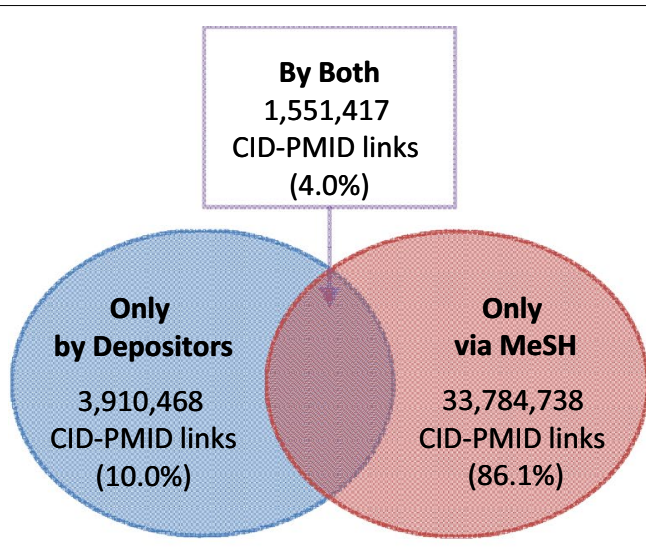

a
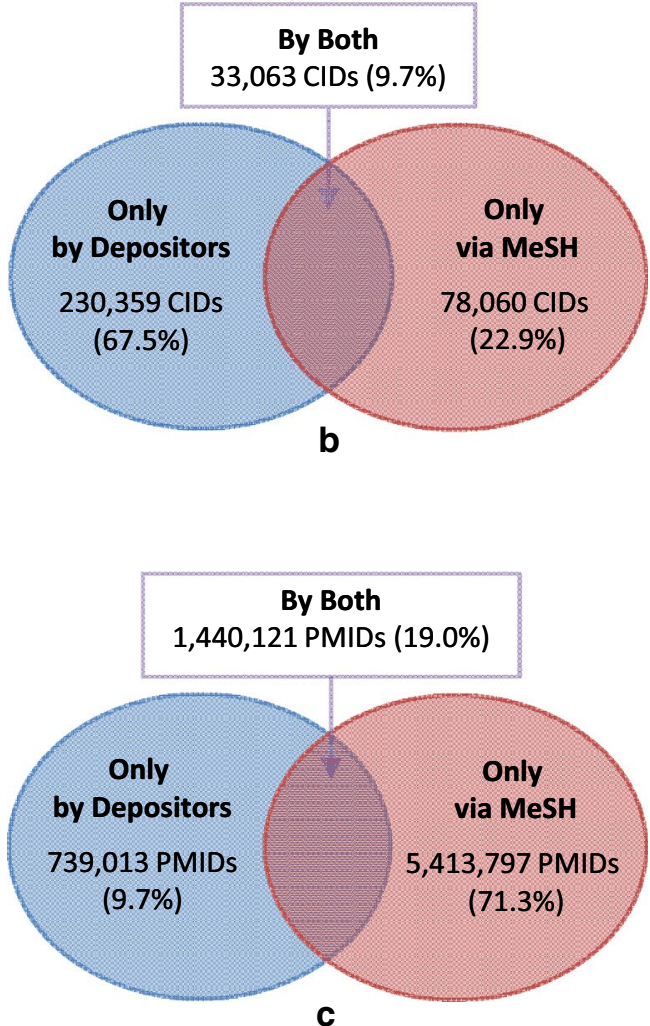

Fig. 7 Venn diagrams for depositor-provided CID-PMID associations and those generated via MeSH. The Venn diagrams compare depositor-provided CID-PMID associations and automated annotations via MeSH in terms of $\mathbf{a}$ the number of CID-PMID associations, and $\mathbf{b}$ CIDs and $\mathbf{c}$ PMIDs involved in these associations

articles that share the same MeSH annotation. This automated process allows PubChem users to leverage the biomedical literature and its $\mathrm{MeSH}$ indexing for search and analysis purposes.

Some journals, such as Nature Chemical Biology, provide PubChem with information on chemicals that appear in their newly published articles. This enables PubChem to direct users to the new articles on the journal web site, even before their abstracts become available in PubMed. In turn, the publisher can provide their readers with access to comprehensive information available in PubChem about the chemicals mentioned in a given article. This exemplifies the mutual benefit of concurrent publication of scientific articles in journals and associated data in public databases.

Literature information, both provided by depositors and derived via $\mathrm{MeSH}$, can be accessed from the DocSum page of an Entrez search result, or from the Compound Summary, Substance Record, or BioAssay Record page. Users can also retrieve PubChem records associated with scientific articles, using appropriate Entrez filters. These tools allow PubChem users to more readily explore information available in literature related to PubChem records.

\section{Authors' contributions}

SK collected and analyzed the data and wrote the first manuscript, and all other authors contributed to writing the manuscript. PAT handles the database infrastructure and access methods. BAS takes care of the data submission system. BY manages the chemical name filtering process. TC, JW, and YW provided and analyzed bioassay-related data. EEB and SHB reviewed the final manuscript. All authors read and approved the final manuscript.

\section{Acknowledgements}

This work was supported by the Intramural Research Program of the National Library of Medicine, National Institutes of Health. Special thanks to PubChem team members (present and past), the Entrez infrastructure group, the MeSH group, and all PubChem data contributors without which this work would not be possible. We also thank Nancy Terry, NIH Library Editing Service, for reviewing the manuscript.

\section{Competing interests}

The authors declare that they have no competing interests.

Received: 15 February 2016 Accepted: 24 May 2016

Published online: 10 June 2016

\section{References}

1. Agarwala R, Barrett T, Beck J, Benson DA, Bollin C, Bolton E, Bourexis D, Brister JR, Bryant SH, Canese K, Clark K, DiCuccio M, Dondoshansky I, Federhen S, Feolo M, Funk K, Geer LY, Gorelenkov V, Hoeppner M, Holmes B, Johnson M, Khotomlianski VE, Kimchi A, Kimelman M, Kitts P, Klimke W, Krasnov S, Kuznetsov A, Landrum MJ, Landsman D et al (2015) Database resources of the National Center for Biotechnology Information. Nucleic Acids Res 43:D6-D17

2. Wang YL, Suzek T, Zhang J, Wang JY, He SQ, Cheng TJ, Shoemaker BA, Gindulyte A, Bryant SH (2014) PubChem BioAssay: 2014 update. Nucleic Acids Res 42:D1075-D1082

3. Wang YL, Bolton E, Dracheva S, Karapetyan K, Shoemaker BA, Suzek TO, Wang JY, Xiao JW, Zhang J, Bryant SH (2010) An overview of the PubChem BioAssay resource. Nucleic Acids Res 38:D255-D266

4. Wang YL, Xiao JW, Suzek TO, Zhang J, Wang JY, Bryant SH (2009) PubChem: a public information system for analyzing bioactivities of small molecules. Nucleic Acids Res 37:W623-W633

5. Bolton EE, Wang Y, Thiessen PA, Bryant SH (2008) PubChem: integrated platform of small molecules and biological activities. In: Ralph AW, David CS (eds) Annual reports in computational chemistry, vol 4. Elsevier, Amsterdam, pp 217-241 
6. Kim S, Thiessen PA, Bolton EE, Chen J, Fu G, Gindulyte A, Han L, He J, He S, Shoemaker BA, Wang J, Yu B, Zhang J, Bryant SH (2016) PubChem substance and compound databases. Nucleic Acids Res 44:D1202-D1213

7. What is the difference between a substance and a compound in PubChem? (2014). https://pubchemblog.ncbi.nlm.nih.gov/2014/06/19/ what-is-the-difference-between-a-substance-and-a-compound-inpubchem/. Accessed 15 April 2016

8. Fact Sheet - PubMed: MEDLINE Retrieval on the World Wide Web (2014) http://www.nlm.nih.gov/pubs/factsheets/pubmed.html. Accessed 15 April 2016

9. PubMed:The Bibliographic Database (2013). http://www.ncbi.nlm.nih. gov/books/NBK153385/. Accessed 15 April 2016

10. PubMed Help [Internet] (2016). http://www.ncbi.nlm.nih.gov/books/ NBK3827/. Accessed 15 April 2016

11. Fact Sheet-MEDLINE, PubMed, and PMC (PubMed Central): How are they different? (2016). http://www.nlm.nih.gov/pubs/factsheets/dif_med_ pub.html. Accessed 15 April 2016

12. Fact Sheet-MEDLINE (2015) http://www.nlm.nih.gov/pubs/factsheets/ medline.html. Accessed 15 April 2016

13. Fact Sheet-Medical Subject Headings (MeSH) (2015) https://www.nlm nih.gov/pubs/factsheets/mesh.html. Accessed 15 April 2016

14. Medical Subject Headings. http://www.ncbi.nlm.nih.gov/mesh. Accessed 15 April 2016

15. Davis AP, Grondin CJ, Lennon-Hopkins K, Saraceni-Richards C, Sciaky D, King BL, Wiegers TC, Mattingly CJ (2015) The comparative toxicogenomics database's 10th year anniversary: update 2015. Nucleic Acids Res 43:D914-D920

16. Southan C, Sharman JL, Benson HE, Faccenda E, Pawson AJ, Alexander SPH, Buneman OP, Davenport AP, McGrath JC, Peters JA, Spedding M, Catterall WA, Fabbro D, Davies JA (2016) NC-IUPHAR: The IUPHAR/BPS Guide to PHARMACOLOGY in 2016: towards curated quantitative interactions between 1300 protein targets and 6000 ligands. Nucleic Acids Res 44:D1054-D1068

17. Wishart DS, Jewison T, Guo AC, Wilson M, Knox C, Liu YF, Djoumbou Y, Mandal R, Aziat F, Dong E, Bouatra S, Sinelnikov I, Arndt D, Xia JG, Liu P, Yallou F, Bjorndahl T, Perez-Pineiro R, Eisner R, Allen F, Neveu V, Greiner R, Scalbert A (2013) HMDB 3.0-the human metabolome database in 2013. Nucleic Acids Res 41:D801-D807

18. Vita R, Overton JA, Greenbaum JA, Ponomarenko J, Clark JD, Cantrell JR, Wheeler DK, Gabbard JL, Hix D, Sette A, Peters B (2015) The immune epitope database (IEDB) 3.0. Nucleic Acids Res 43:D405-D412

19. Caspi R, Altman T, Billington R, Dreher K, Foerster H, Fulcher CA, Holland TA, Keseler IM, Kothari A, Kubo A, Krummenacker M, Latendresse M,
Mueller LA, Ong Q, Paley S, Subhraveti P, Weaver DS, Weerasinghe D, Zhang PF, Karp PD (2014) The MetaCyc database of metabolic pathways and enzymes and the BioCyc collection of Pathway/Genome Databases. Nucleic Acids Res 42:D459-D471

20. Law V, Knox C, Djoumbou Y, Jewison T, Guo AC, Liu YF, Maciejewski A, Arndt D, Wilson M, Neveu V, Tang A, Gabriel G, Ly C, Adamjee S, Dame ZT, Han BS, Zhou Y, Wishart DS (2014) DrugBank 4.0: shedding new light on drug metabolism. Nucleic Acids Res 42:D1091-D1097

21. Gao JF, Ellis LBM, Wackett LP (2010) The University of Minnesota biocatalysis/biodegradation database: improving public access. Nucleic Acids Res 38:D488-D491

22. Bento AP, Gaulton A, Hersey A, Bellis LJ, Chambers J, Davies M, Kruger FA, Light Y, Mak L, McGlinchey S, Nowotka M, Papadatos G, Santos R, Overington JP (2014) The ChEMBL bioactivity database: an update. Nucleic Acids Res 42:D1083-D1090

23. Liu ZH, Li Y, Han L, Li J, Liu J, Zhao ZX, Nie W, Liu YC, Wang RX (2015) PDBwide collection of binding data: current status of the PDBbind database. Bioinformatics 31:405-412

24. Berman HM, Westbrook J, Feng Z, Gilliland G, Bhat TN, Weissig H, Shindyalov IN, Bourne PE (2000) The protein data bank. Nucleic Acids Res 28:235-242

25. Madej T, Lanczycki CJ, Zhang DC, Thiessen PA, Geer RC, Marchler-Bauer A, Bryant SH (2014) MMDB and VAST+: tracking structural similarities between macromolecular complexes. Nucleic Acids Res 42:D297-D303

26. Liu TQ, Lin YM, Wen X, Jorissen RN, Gilson MK (2007) BindingDB: a webaccessible database of experimentally determined protein-ligand binding affinities. Nucleic Acids Res 35:D198-D201

27. Okuno Y, Tamon A, Yabuuchi H, Niijima S, Minowa Y, Tonomura K, Kunimoto R, Feng CL (2008) GLIDA: GPCR — ligand database for chemical genomics drug discovery-database and tools update. Nucleic Acids Res 36:D907-D912

28. Schuler GD, Epstein JA, Ohkawa H, Kans JA (1996) Entrez: molecular biology database and retrieval system. Methods Enzymol 266:141-162

29. McEntyre J (1998) Linking up with Entrez. Trends Genet 14:39-40

30. Entrez Programming Utilities Help. http://www.ncbi.nlm.nih.gov/books/ NBK25501

31. Kim S, Thiessen PA, Bolton EE, Bryant SH (2015) PUG-SOAP and PUGREST: web services for programmatic access to chemical information in PubChem. Nucleic Acids Res 43:W605-W611

\section{Submit your manuscript to a SpringerOpen ${ }^{\circ}$ journal and benefit from:}

- Convenient online submission

- Rigorous peer review

- Immediate publication on acceptance

- Open access: articles freely available online

- High visibility within the field

- Retaining the copyright to your article

Submit your next manuscript at springeropen.com 